\title{
Populations of Phytophthora infestans in Israel Underwent Three Major Genetic Changes During 1983 to 2000
}

\author{
Yigal Cohen
}

Faculty of Life Sciences, Bar-Ilan University, Ramat-Gan 52900, Israel.

Accepted for publication 6 November 2001.

\section{ABSTRACT}

Cohen, Y. 2002. Populations of Phytophthora infestans in Israel underwent three major genetic changes during 1983 to 2000. Phytopathology 92:300-307.

In this survey, 799 isolates of Phytophthora infestans collected from potato crops in Israel during 1983 to 2000 were analyzed for mating type and sensitivity to metalaxyl, and 324 were analyzed for race structure. The $\mathrm{A}_{2}$ mating type, first recorded in 1983, fully dominated the pathogen population from 1983 until 1991 (9 years). It was thereafter replaced by the $A_{1}$ mating type, which dominated the population during 1993 to 2000. Metalaxyl-resistant isolates were first recorded in 1982. During 1983 to 1991 , the majority of the isolates were resistant. Isolates with intermediate sensitivity (I) to this fungicide were first observed in 1993, when both $A_{1}$ and $A_{2}$ mating types occurred in the population. The proportion of I isolates gradually increased, reaching 39 to $41 \%$ in 1997 to 1998 , and then declined to $\approx 15 \%$ in 1999 to 2000 . Pathogenicity to nine potato differential cultivars was determined for 80 potato isolates collected in 1983 to 1991 , to 11 potato differentials in 173 isolates collected in 1993 to 1998, and in 71 potato isolates collected in 1999 to 2000. The first population was composed of 5 races with race 1,3,4,7,8,10 predominating $(76 \%)$, the second population was composed of 19 races with race $1,3,4,7,8,10,11$ predominating $(63 \%)$, and the third population exhibited 42 (34 new) races with no single predominating race. RG-57 DNA fingerprinting and allozymes loci assays of 23 isolates revealed that isolates collected during 1984 to 1986 belonged to the PO-57 lineage, whereas those collected during 1997 to 1999 belonged to the RFO-39 lineage. Among isolates collected during 1993 to 1995, two unreported DNA fingerprinting patterns were found. Severe late blight epidemics occurred in tomato crops during 1998 to 2000 . Of 35 tomato isolates, 28 were $A_{1}$ and only 7 were $\mathrm{A}_{2}$. Of these tomato isolates, $94 \%$ were sensitive to metalaxyl. Almost every isolate had a different race structure on the 11 potato differentials. When inoculated onto three tomato differential cultivars, tomato isolates showed a virulence much more enhanced than potato isolates. The data suggest the Israeli population of $P$. infestans has passed through three major genetic changes during the past 18 years: in 1983, 1993, and 1999. The recent change included host specialization to tomato.

Additional keywords: epidemiology, oospores.
Late blight caused by the oomycete pathogen Phytophthora infestans is a devastating disease of potato and tomato worldwide (1). Severe epidemics of late blight occur in potato in Israel in both the autumn-planted crops and the spring-planted crops, especially under rainy conditions. In recent years, disease has become more severe, especially in tomato crops. Tomatoes are grown all year around, especially in net and plastic houses. $P$. infestans is heterothallic, requiring both $\mathrm{A}_{1}$ and $\mathrm{A}_{2}$ mating types for the production of sexual oospores. While sporangia, produced vegetatively, are the sole source of inoculum during the epidemic phase of the disease, oospores produced by mating in the field (2) may serve as an overwintering soilborne inoculum $(1,33)$. Oospores are not only a long-lasting source of inoculum but also may supply new recombinant genotypes of the pathogen $(6,7,38)$, with possibly altered virulence, host specialization $(10,16,28)$, and response to phenylamide fungicides (e.g., metalaxyl) (11).

$\mathrm{A}_{2}$ mating type isolates were first detected in Israel in 1983 (17), a few years after being recorded in Europe (18). In a previous survey in Israel by Grinberger et al. (17) during 1983 to 1989 , it was found that $\mathrm{A}_{2}$ isolates predominated the fungal population; that is, 37 out of 38 potato isolates collected from an area of $\approx 800 \mathrm{~km}^{2}$ belonged to the $\mathrm{A}_{2}$ mating type. Another survey conducted in my laboratory by Kadish (20) during 1983 to 1991 revealed that out of 544 single-lesion isolates, $69 \%$ were resistant to metalaxyl. Earlier studies made during 1954 to 1970 in Israel

Corresponding author: Y. Cohen; E-mail address: coheny@mail.biu.ac.il

Publication no. P-2002-0122-01R

(C) 2002 The American Phytopathological Society showed the occurrence of races $0,1,3,4,1.4$, and 1.3.4, with race 4 being most frequent $(25,31,36)$. Race structure analysis conducted in 1995 for isolates collected from potato (11) showed that about half of the isolates carried the virulence factors 1.3.4.7.8.10.11.

The aim of the present survey was to monitor some of the genetic changes (mating type, race structure, and sensitivity to metalaxyl) in the population of $P$. infestans in commercial potato and tomato crops and to possibly relate these changes to the severe epidemics recently caused by the pathogen in nature.

\section{MATERIALS AND METHODS}

Sample collection. This survey reports on samples collected during October 1983 to December 2000 from commercial fields distributed over an area of $\approx 3,000 \mathrm{~km}^{2}$, from Bet-She' an County in the northeast to the Bsor County in the southwest. Blighted foliage of potato or tomato were collected by us or county agents and shipped overnight to the laboratory at Bar-Ilan University. Infected leaves were placed in moistened petri dishes at $15^{\circ} \mathrm{C}$ for $20 \mathrm{~h}$ in the dark to enhance sporulation of the pathogen. Sporangia were collected from a single leaflet into ice-cold glass-distilled water and used for inoculation. Samples were collected during the epidemic season, namely October through May. No samples were obtained during June through September except for a single case in July from tomato.

Bioassays. Sporangia from each sample (isolate) were used for three bioassays: mating type determination, compatibility with differential cultivars (virulence phenotype), and sensitivity to metalaxyl. Mating type assay was conducted as described by Cohen et al. (3). Sensitivity to metalaxyl was conducted as described by Kadish et al. (21). Pathogenicity assays were done with 
the 12 standard potato differentials, $\mathrm{R}_{0}$ to $\mathrm{R}_{11}$, obtained from $\mathrm{U}$. Gisi (Syngenta Crop Protection, Stein, Switzerland). Plants were clonally reproduced in vitro from stem segments and then grown in 2-liter pots in the greenhouse. Leaflets were detached, placed in moistened petri dishes, lower side uppermost, and inoculated with six 10- $\mu$ l droplets per leaflet (5,000 sporangia per ml), two leaflets per potato genotype. Plates were incubated in $15^{\circ} \mathrm{C}$ growth chambers with a 12 -h photoperiod for 10 days. Reaction to the pathogen was assessed as either compatible (expanded lesions with sporulation) or incompatible (hypersensitive response). Virulence factors of an isolate are given as a series of numbers, representing the R-gene potato genotypes that produce compatible interaction with that isolate. In all tests, tomato leaflets (inbred $\mathrm{ZH}$ or cv. Baby carrying no resistance genes) were also included. For some isolates compatibility with three tomato genotypes was also determined: $\mathrm{ZH}$ (own inbred, no genes for resistance, $P h-0$ ), NewYorker (carrying $P h-1$ ), and Pieraline (carrying $P h-2$ ). The following index was used to visually assess compatibility: $\mathrm{HR}=$ hypersensitive response, dark-brown lesions with no sporulation; $1=$ lesions, 5 to $10 \mathrm{~mm}$ in diameter, with scarce sporulation; $2=$ lesions, 10 to $15 \mathrm{~mm}$ in diameter, with moderate sporulation; $3=$ lesions, 15 to $20 \mathrm{~mm}$ in diameter, with abundant sporulation; and 4 = lesions, 20 to $25 \mathrm{~mm}$ in diameter, with abundant sporulation.

Allozymes and DNA fingerprinting. A subset of 23 isolates of $P$. infestans were grown in liquid rye medium. Mycelia were used for allozymes loci assays as described by Goodwin et al. (15) and for analyzing nuclear DNA fingerprint by the highly polymorphic, moderately repetitive DNA probe RG-57 (14). The octal nomenclature system was used to summarize the DNA banding data as described by Goodwin et al. (14).

\section{RESULTS}

Mating type. In all, 799 isolates obtained from commercial potato fields during 1983 to 2000 (except 1992) were analyzed for mating type (Fig. 1A) and sensitivity to metalaxyl (Fig. 1B). From its appearance in 1983 until 1991 ( 9 years), the $A_{2}$ mating type dominated the population of $P$. infestans in the country (Fig. 1A), composing 98 to $100 \%$ of all isolates collected (547 isolates). A sharp decline in the proportion of the $\mathrm{A}_{2}$ mating type (to 29\%) occurred in 1993 (no data available for 1992). This decline continued until 1996, when no $\mathrm{A}_{2}$ isolates were detected in the population. In 1997 to 2000, the frequency of $\mathrm{A}_{2}$ remained very low (0 to 4\%) except for 1999 (28\%). Thirteen isolates collected during January to April 2001 were all $\mathrm{A}_{1}$ (data not shown).

Sensitivity to metalaxyl. The fungal population during 1983 to 1991 (9 years) was composed of either sensitive (S) or resistant (R) isolates (Fig. 1B). In 7 out of 9 years, $\mathrm{R}$ isolates predominated (58 to $83 \%$ ) in the population; whereas, in the other 2 years (1984 and 1985), approximately equal proportions of S and R occurred. In 1993, two events took place: isolates with intermediate (I) resistance to metalaxyl were detected $(14 \%)$ for the first time and the proportion of $\mathrm{R}$ declined (from $72 \%$ in 1991) to $43 \%$. The frequency of I isolates increased until 1998 (41\%) and declined toward 2000. The frequency of $\mathrm{R}$ isolates continued to drop until 1995 to its lowest level (7\%), and then reached another peak (57\%) in 1997. During 1999 to $2000, \mathrm{~S}$ isolates composed $\approx 60 \%$ of the population and I and $\mathrm{R}$ isolates $\approx 20 \%$ each. Of 13 isolates collected during January to April 2001, 6 were S and 7 were I (data not shown), with no $\mathrm{R}$ isolates detected.

Severe epiphytotics of $P$. infestans occurred in tomato crops during 1998 to 2000. Seven isolates were collected from tomato in 1998. All were sensitive to metalaxyl, 5 belonged to $A_{1}$, and 2 belonged to $\mathrm{A}_{2}$ mating type. Out of 18 isolates collected in 1999, 13 were $A_{1}$ and 5 were $A_{2}$, with 16,1 , and 1 showing an $S$, I, and $\mathrm{R}$ response, respectively, to metalaxyl. In 2000, 10 isolates were collected; all were $\mathrm{A}_{1}$ and sensitive to metalaxyl. Two isolates collected in January 2001 were also $A_{1}$ and $S$ (data not shown).
Race structure (virulence phenotypes) of potato isolates. Whereas most isolates collected during 1993 to 2000 were tested for virulence factors, only a small number of the isolates collected during 1983 to 1991 were similarly tested. The race structure of 324 potato isolates collected during 1983 to 2000 is shown in Table 1. Data for 1983 to 1986 and 1987 to 1991 are taken from Kadish and Cohen (23) and Kadish (20), respectively. Data for each of these two periods were pooled together because year-byyear analyses were not available. No data are available for 1992. The compatibility or incompatibility of the isolates collected during 1993 to 2000 was tested on the $R_{0}$ to $R_{11}$ potato differential lines, whereas that of the isolates collected during 1983 to 1991 was tested on only nine of the potato differentials (excluding $\mathrm{R}_{5}, \mathrm{R}_{6}$, and $\mathrm{R}_{11}$ ).
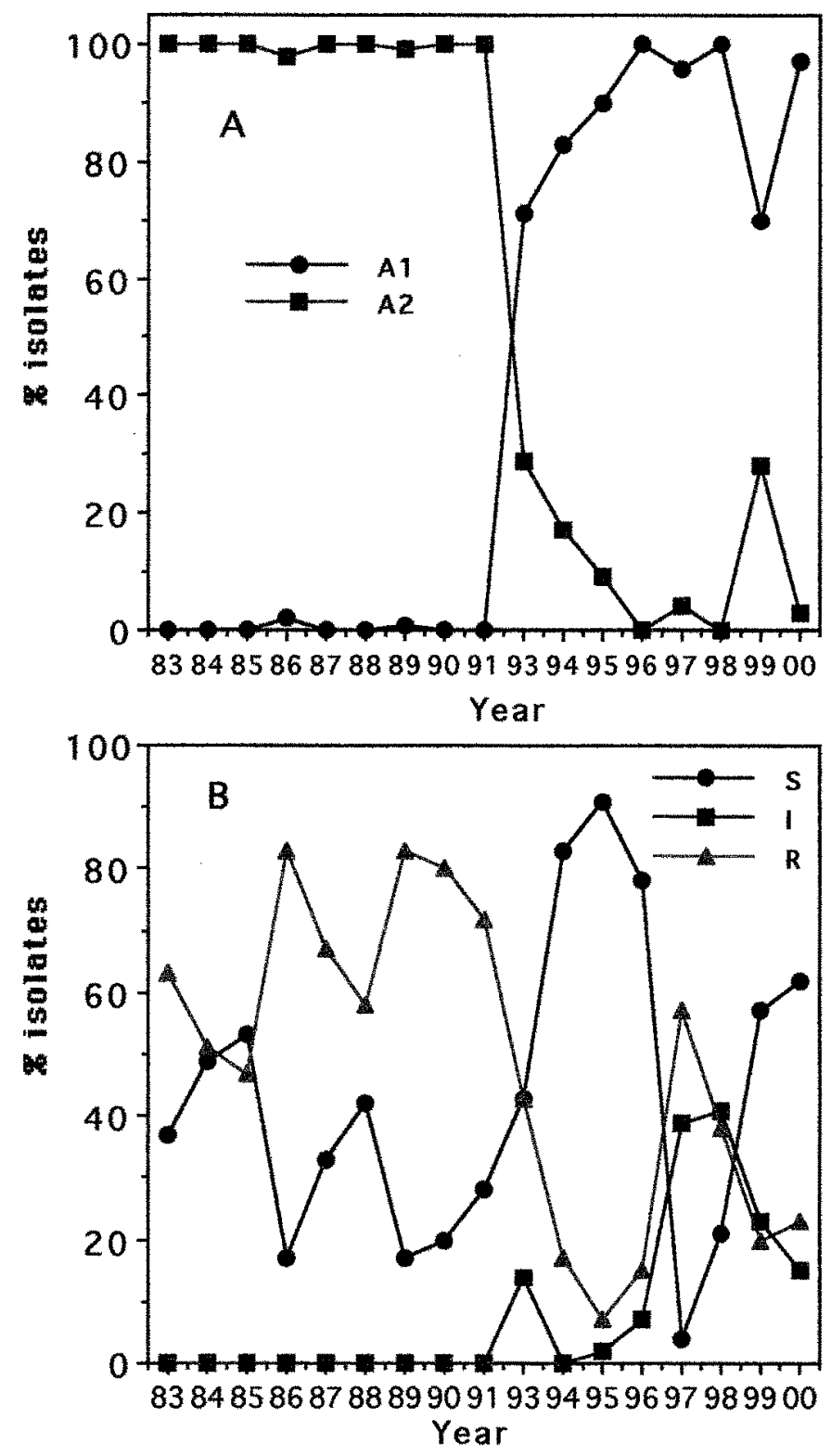

43673048397372859076584528294039

\section{Number of isolates}

Fig. 1. Genetic changes in potato isolates of Phytophthora infestans during 1983 to 2000. A, Frequency of $A_{1}$ and $A_{2}$ mating types among 799 isolates. Data for 1983 to 1988 are from Grinberger et al. (17). B, Frequency of metalaxyl-sensitive isolates (S), metalaxyl-resistant isolates (R), and isolates with intermediate (I) response to metalaxyl among 799 isolates. Data for 1983 to 1991 are from Kadish and Cohen (23). 
Only six isolates were tested for 1983 to 1986 . Three belonged to race $1,3,4,7,8$ and three to race $1,3,4,7,8,10$. Among the 74 isolates collected during 1987 to 1991 , only five races were detected, with $78 \%$ being $1,3,4,7,8,10$ and $9 \%$ race $1,3,4,7,8$. The population collected during 1993 to 1998 was mostly composed of race $1,3,4,7,8,10,11$ (112 of 173 isolates, 63\%). In 1999, a major change occurred in the race structure of the population: the frequency of race $1,3,4,7,8,10,11$, which predominated during 1993

TABLE 1. Race structure of Phytophthora infestans isolates collected from potato crops during 1983 to 2000

\begin{tabular}{|c|c|c|c|c|c|c|c|c|c|c|c|}
\hline \multirow[b]{2}{*}{ Pathotype no., structure ${ }^{b}$} & \multicolumn{10}{|c|}{ Years $^{\mathrm{a}}$} & \multirow[b]{2}{*}{ Total } \\
\hline & $1983-86$ & 1987-91 & 1993 & 1994 & 1995 & 1996 & 1997 & 1998 & 1999 & 2000 & \\
\hline \multicolumn{12}{|l|}{3} \\
\hline 134 & $\ldots$ & 1 & $\ldots$ & $\ldots$ & $\ldots$ & $\ldots$ & $\ldots$ & $\ldots$ & $\ldots$ & 1 & 2 \\
\hline \multicolumn{12}{|l|}{4} \\
\hline 1347 & $\ldots$ & 2 & 1 & $\ldots$ & 2 & $\ldots$ & $\ldots$ & $\ldots$ & 2 & $\ldots$ & 7 \\
\hline 1349 & $\ldots$ & $\ldots$ & $\ldots$ & $\ldots$ & $\ldots$ & $\ldots$ & $\ldots$ & $\ldots$ & $\ldots$ & 2 & 2 \\
\hline 13411 & $\ldots$ & $\ldots$ & $\ldots$ & $\ldots$ & $\ldots$ & $\ldots$ & $\ldots$ & $\ldots$ & 1 & $\ldots$ & 1 \\
\hline 13711 & $\ldots$ & $\ldots$ & $\ldots$ & $\ldots$ & $\ldots$ & $\ldots$ & $\ldots$ & $\ldots$ & 1 & $\ldots$ & 1 \\
\hline \multicolumn{12}{|l|}{5} \\
\hline 13457 & $\ldots$ & $\ldots$ & $\ldots$ & $\ldots$ & $\ldots$ & $\ldots$ & $\ldots$ & $\ldots$ & 2 & $\ldots$ & 2 \\
\hline 13458 & $\ldots$ & $\ldots$ & $\ldots$ & $\ldots$ & $\ldots$ & $\ldots$ & $\ldots$ & $\ldots$ & 1 & $\ldots$ & 1 \\
\hline 134511 & $\ldots$ & $\ldots$ & $\ldots$ & $\ldots$ & $\ldots$ & $\ldots$ & $\ldots$ & $\ldots$ & 1 & $\ldots$ & 1 \\
\hline 13467 & $\ldots$ & $\ldots$ & $\ldots$ & 1 & 2 & $\ldots$ & $\ldots$ & $\ldots$ & $\ldots$ & $\ldots$ & 3 \\
\hline 13478 & 3 & 7 & $\ldots$ & 1 & 4 & 2 & 1 & $\ldots$ & $\ldots$ & $\ldots$ & 18 \\
\hline 13479 & $\ldots$ & $\ldots$ & $\ldots$ & $\ldots$ & $\ldots$ & $\ldots$ & $\ldots$ & $\ldots$ & $\ldots$ & 3 & 3 \\
\hline 134710 & $\ldots$ & 6 & $\ldots$ & $\ldots$ & $\ldots$ & $\ldots$ & $\ldots$ & $\ldots$ & 1 & $\ldots$ & 7 \\
\hline 134711 & $\ldots$ & $\ldots$ & 1 & $\ldots$ & 1 & $\ldots$ & $\ldots$ & $\ldots$ & 2 & $\ldots$ & 4 \\
\hline 13489 & $\ldots$ & $\ldots$ & $\ldots$ & $\ldots$ & $\ldots$ & $\ldots$ & $\ldots$ & $\ldots$ & $\ldots$ & 1 & 1 \\
\hline 147911 & $\ldots$ & $\ldots$ & $\ldots$ & $\ldots$ & $\ldots$ & $\ldots$ & $\ldots$ & $\ldots$ & 1 & $\ldots$ & 1 \\
\hline 1471011 & $\ldots$ & $\ldots$ & $\ldots$ & $\ldots$ & $\ldots$ & $\ldots$ & $\ldots$ & $\ldots$ & 1 & $\ldots$ & 1 \\
\hline 16789 & $\ldots$ & $\ldots$ & $\ldots$ & $\ldots$ & $\ldots$ & $\ldots$ & $\ldots$ & $\ldots$ & 1 & $\ldots$ & 1 \\
\hline \multicolumn{12}{|l|}{6} \\
\hline 1234811 & $\ldots$ & $\ldots$ & $\ldots$ & $\ldots$ & $\ldots$ & $\ldots$ & $\ldots$ & $\ldots$ & $\ldots$ & 1 & 1 \\
\hline 134567 & $\ldots$ & $\ldots$ & $\ldots$ & $\ldots$ & 1 & $\ldots$ & $\ldots$ & $\ldots$ & $\ldots$ & $\ldots$ & 1 \\
\hline 1345710 & $\ldots$ & $\ldots$ & $\ldots$ & $\ldots$ & $\ldots$ & $\ldots$ & $\ldots$ & $\ldots$ & 2 & $\ldots$ & 2 \\
\hline 1345711 & $\ldots$ & $\ldots$ & $\ldots$ & $\ldots$ & $\ldots$ & $\ldots$ & $\ldots$ & $\ldots$ & 3 & $\ldots$ & 3 \\
\hline 134678 & $\ldots$ & $\ldots$ & 1 & $\ldots$ & 1 & $\ldots$ & $\ldots$ & $\ldots$ & $\ldots$ & $\ldots$ & 2 \\
\hline 134679 & $\ldots$ & $\ldots$ & $\ldots$ & $\ldots$ & $\ldots$ & $\ldots$ & $\ldots$ & $\ldots$ & $\ldots$ & 1 & 1 \\
\hline 1346710 & $\ldots$ & $\ldots$ & 1 & $\ldots$ & $\ldots$ & $\ldots$ & $\ldots$ & $\ldots$ & $\ldots$ & $\ldots$ & 1 \\
\hline 1346711 & $\ldots$ & $\ldots$ & $\ldots$ & $\ldots$ & 1 & $\ldots$ & $\ldots$ & $\ldots$ & $\ldots$ & $\ldots$ & 1 \\
\hline 1347810 & 3 & 58 & $\ldots$ & $\ldots$ & $\ldots$ & $\ldots$ & $\ldots$ & $\ldots$ & $\ldots$ & $\ldots$ & 61 \\
\hline 1347811 & $\ldots$ & $\ldots$ & 2 & 1 & 6 & $\ldots$ & $\ldots$ & $\ldots$ & $\ldots$ & $\ldots$ & 9 \\
\hline 13471011 & $\ldots$ & $\ldots$ & $\ldots$ & $\ldots$ & 5 & 1 & $\ldots$ & $\ldots$ & 7 & $\ldots$ & 13 \\
\hline 1348911 & $\ldots$ & $\ldots$ & $\ldots$ & $\ldots$ & $\ldots$ & $\ldots$ & $\ldots$ & $\ldots$ & $\ldots$ & 1 & 1 \\
\hline 13491011 & $\ldots$ & $\ldots$ & $\ldots$ & $\ldots$ & $\ldots$ & $\ldots$ & $\ldots$ & $\ldots$ & $\ldots$ & 3 & 3 \\
\hline 145679 & $\ldots$ & $\ldots$ & $\ldots$ & $\ldots$ & $\ldots$ & $\ldots$ & $\ldots$ & $\ldots$ & $\ldots$ & 1 & 1 \\
\hline \multicolumn{12}{|l|}{7} \\
\hline 12347811 & $\ldots$ & $\ldots$ & $\ldots$ & $\ldots$ & 1 & $\ldots$ & $\ldots$ & $\ldots$ & $\ldots$ & $\ldots$ & 1 \\
\hline 1246789 & $\ldots$ & $\ldots$ & $\ldots$ & $\ldots$ & $\ldots$ & $\ldots$ & $\ldots$ & $\ldots$ & 1 & $\ldots$ & 1 \\
\hline 1345679 & $\ldots$ & $\ldots$ & $\ldots$ & $\ldots$ & $\ldots$ & $\ldots$ & $\ldots$ & $\ldots$ & $\ldots$ & 2 & 2 \\
\hline 134571011 & $\ldots$ & $\ldots$ & $\ldots$ & $\ldots$ & $\ldots$ & $\ldots$ & $\ldots$ & $\ldots$ & 3 & $\ldots$ & 3 \\
\hline 13467810 & $\ldots$ & $\ldots$ & $\ldots$ & 1 & $\ldots$ & $\ldots$ & $\ldots$ & $\ldots$ & $\ldots$ & $\ldots$ & 1 \\
\hline 13467811 & $\ldots$ & $\ldots$ & $\ldots$ & 1 & 1 & $\ldots$ & $\ldots$ & $\ldots$ & $\ldots$ & $\ldots$ & 2 \\
\hline 13467911 & $\ldots$ & $\ldots$ & $\ldots$ & $\ldots$ & $\ldots$ & $\ldots$ & $\ldots$ & $\ldots$ & 1 & $\ldots$ & 1 \\
\hline 134681011 & $\ldots$ & $\ldots$ & $\ldots$ & $\ldots$ & 1 & $\ldots$ & $\ldots$ & $\ldots$ & $\ldots$ & $\ldots$ & 1 \\
\hline 13478911 & $\ldots$ & $\ldots$ & $\ldots$ & $\ldots$ & $\ldots$ & $\ldots$ & $\ldots$ & $\ldots$ & $\ldots$ & 2 & 2 \\
\hline 134781011 & $\ldots$ & $\ldots$ & 1 & 1 & 26 & 34 & 19 & 28 & 3 & $\ldots$ & 112 \\
\hline 134791011 & $\ldots$ & $\ldots$ & $\ldots$ & $\ldots$ & $\ldots$ & $\ldots$ & $\ldots$ & $\ldots$ & 1 & $\ldots$ & 1 \\
\hline 134891011 & $\ldots$ & $\ldots$ & $\ldots$ & $\ldots$ & $\ldots$ & $\ldots$ & $\ldots$ & $\ldots$ & $\ldots$ & 2 & 2 \\
\hline 137891011 & $\ldots$ & $\ldots$ & $\ldots$ & $\ldots$ & $\ldots$ & $\ldots$ & $\ldots$ & $\ldots$ & $\ldots$ & 1 & 1 \\
\hline 146781011 & $\ldots$ & $\ldots$ & $\ldots$ & $\ldots$ & $\ldots$ & $\ldots$ & 1 & $\ldots$ & $\ldots$ & $\ldots$ & 1 \\
\hline 167891011 & $\ldots$ & $\ldots$ & $\ldots$ & $\ldots$ & $\ldots$ & $\ldots$ & $\ldots$ & $\ldots$ & $\ldots$ & 1 & 1 \\
\hline \multicolumn{12}{|l|}{8} \\
\hline 123467911 & $\ldots$ & $\ldots$ & $\ldots$ & $\ldots$ & $\ldots$ & $\ldots$ & $\ldots$ & $\ldots$ & $\ldots$ & 1 & 1 \\
\hline 1234671011 & $\ldots$ & $\ldots$ & $\ldots$ & $\ldots$ & 1 & $\ldots$ & $\ldots$ & $\ldots$ & $\ldots$ & $\ldots$ & 1 \\
\hline 123478910 & $\ldots$ & $\ldots$ & $\ldots$ & $\ldots$ & $\ldots$ & $\ldots$ & $\ldots$ & $\ldots$ & 1 & $\ldots$ & 1 \\
\hline 13456789 & $\ldots$ & $\ldots$ & $\ldots$ & $\ldots$ & $\ldots$ & $\ldots$ & $\ldots$ & $\ldots$ & $\ldots$ & 1 & 1 \\
\hline 1345781011 & $\ldots$ & $\ldots$ & $\ldots$ & $\ldots$ & $\ldots$ & $\ldots$ & $\ldots$ & $\ldots$ & 1 & $\ldots$ & 1 \\
\hline 1346781011 & $\ldots$ & $\ldots$ & $\ldots$ & $\ldots$ & 4 & 8 & 7 & 1 & 1 & $\ldots$ & 21 \\
\hline 1347891011 & $\ldots$ & $\ldots$ & $\ldots$ & $\ldots$ & $\ldots$ & $\ldots$ & $\ldots$ & $\ldots$ & 1 & 2 & 3 \\
\hline 9 & & & & & & & & & & & \\
\hline 1234678911 & $\ldots$ & $\ldots$ & $\ldots$ & $\ldots$ & $\ldots$ & $\ldots$ & $\ldots$ & $\ldots$ & $\ldots$ & 1 & 1 \\
\hline 12346781011 & $\ldots$ & $\ldots$ & $\ldots$ & $\ldots$ & 1 & $\ldots$ & $\ldots$ & $\ldots$ & $\ldots$ & 1 & 2 \\
\hline 12347891011 & $\ldots$ & $\ldots$ & $\ldots$ & $\ldots$ & $\ldots$ & $\ldots$ & $\ldots$ & $\ldots$ & $\ldots$ & 4 & 4 \\
\hline Isolates per year (period) & 6 & 74 & 7 & 6 & 58 & 45 & 28 & 29 & 39 & 32 & 324 \\
\hline Virulence phenotypes & 2 & 5 & 6 & 6 & 16 & 4 & 4 & 2 & 23 & 20 & $\ldots$ \\
\hline Isolates/phenotype & $\ldots$ & 14.8 & $\ldots$ & $\ldots$ & 3.6 & 11.3 & 7.0 & 14.5 & 1.7 & 1.6 & $\ldots$ \\
\hline
\end{tabular}

a Data for 1983 to 1986 were taken from Kadish and Cohen (23) and for 1987 to 1991 from Kadish (20).

b Virulence phenotype number and structure. 
to 1998 (6 years), sharply declined (to $8 \%$ ) and 17 new races appeared, indicating the evolution of a new population. Furthermore, the population of 2000 was composed of 20 races, of which 17 were new (not detected in the past 17 years, 1983 to 1999). Race $1,3,4,7,8,10,11$ was not detected in 2000 nor in the additional 13 isolates collected during January to April 2001 (data not shown). Almost every second isolate collected in 1999 to 2000 belonged to a different race, whereas 3.6 to 14.8 isolates per race were found in the populations of 1983 to 1998 (Table 1).

The 324 isolates of 1983 to 2000 were grouped according to the number of virulence factors they contained (and number of combinations), their mating type $\left(\mathrm{A}_{1}\right.$ or $\left.\mathrm{A}_{2}\right)$, and response to metalaxyl (S, I, or R) (Table 2).

The 1983 to 1991 population (80 isolates) carried three to six virulence factors which were added to only five combinations. Of these isolates, $\approx 73 \%$ carried six virulence factors. Mean number of virulence factors per isolate was 5.68. All isolates were $\mathrm{A}_{2}$ and segregated $\approx 1: 1$ into sensitive or resistant to metalaxyl.

The 1993 to 1998 population (173 isolates) carried four to nine virulence factors in 19 different combinations. The majority of the isolates $(66.5 \%)$ carried seven virulence factors and only one isolate carried nine factors. Mean number of virulence factors per isolate was 6.82 . The vast majority of the isolates belonged to the $A_{1}$ mating type $(95 \%)$. Of these, $\approx 60,16$, and $24 \%$ were $S$, I, and $\mathrm{R}$, respectively, to metalaxyl. No $\mathrm{A}_{2} \mathrm{I}$ isolates were detected in this population.

The 1999 to 2000 population (71 isolates) had three to nine virulence factors which were combined into as many as 42 races, with approximately every second isolate presenting a different race (Table 1). The mean number of virulence factors per isolate was 6.31. Of the isolates, $\approx 80 \%$ belonged to the $\mathrm{A}_{1}$ mating type, with $\approx 55,20$, and $25 \%$ responding as $\mathrm{S}, \mathrm{I}$, and $\mathrm{R}$, respectively, to metalaxyl. Interestingly, only 11 of these 42 races also were detected in the tomato population, meaning that 31 races were specific to potato.

Data collected during 1987 to 1991 showed that potato tubers imported to Israel from Europe carried 11, mostly simple, races of $P$. infestans (20). Out of 29 isolates collected (14 sensitive and 15 resistant to metalaxyl), 22 represented five races and carried two to three virulence factors, 6 had four to five factors, and only 1 had the race structure $1,3,4,7,8,10$ (20).

Comparative data on the frequency of appearance of the 11 virulence factors in the three populations collected from potato during 1983 to 1991,1993 to 1998 , and 1999 to 2000 is shown in Table 3 . The frequency of virulence factors 2, 5, and 9 increased, whereas that of 7, 8, 10, and 11 decreased.

DNA-fingerprinting and allozymes of potato isolates. Twenty-three isolates were examined for fingerprinting with the DNA RG-57 probe (14) and allozymes loci of peptidase (Pep) and glucose-1,6-phospho-isomerase (Gpi) (15). Nine isolates collected during 1984 to 1986 belonged to the PO-57 lineage found in Poland and Russia $(8,13)$ (Table 4). Four isolates sampled during 1993 to 1995 retained their allozymes loci but three exhibited two different patterns of RG-57 banding which do not occur elsewhere (8). Still another change occurred in the 10 isolates collected during 1997 to 1999 . They all belonged to lineage RFO-39 found in the United Kingdom and Western Europe (D. Shaw, personal communication).

Race structure of tomato isolates. Late blight in tomato was a minor disease in Israel until recently. Two $A_{1}$ isolates were obtained in 1997, and five $A_{1}$ and two $A_{2}$ isolates in 1998. In 1999 and 2000, severe epiphytotics developed in tomato crops grown in plastic houses and net houses, especially in the northwestern Negev (Bsor County). Of the 28 isolates collected during 1999 to 2000, 23 belonged to the $A_{1}$ and 5 to the $A_{2}$ mating type (Table 5). All except two isolates from tomato were sensitive to metalaxyl

TABLE 3. The frequency of appearance of 11 virulence factors in potato and tomato isolates of Phytophthora infestans collected during 1983 to 2000

\begin{tabular}{|c|c|c|c|c|}
\hline \multirow[b]{3}{*}{$\begin{array}{l}\text { Virulence } \\
\text { factor }\end{array}$} & \multicolumn{4}{|c|}{ Frequency of appearance $(\%)^{\mathrm{a}}$} \\
\hline & \multicolumn{3}{|c|}{ Potato } & \multirow{2}{*}{$\begin{array}{c}\text { Tomato } \\
1998-2000 \\
(n=32)\end{array}$} \\
\hline & $\begin{array}{c}1983-1991 \\
(n=80)\end{array}$ & $\begin{array}{c}1993-1998 \\
(n=173)\end{array}$ & $\begin{array}{c}1999-2000 \\
(n=71)\end{array}$ & \\
\hline 1 & 100 & 100 & 100 & 100 \\
\hline 2 & 0 & 1.7 & 12.3 & 15.6 \\
\hline 3 & 100 & 99.4 & 86.3 & 93.8 \\
\hline 4 & 100 & 100 & 89 & 90.6 \\
\hline 5 & $\mathrm{nt}$ & 0.6 & 23.3 & 21.9 \\
\hline 6 & nt & 20.2 & 17.8 & 25.0 \\
\hline 7 & 98.8 & 99.4 & 65.7 & 84.3 \\
\hline 8 & 74.4 & 89.6 & 39.7 & 15.6 \\
\hline 9 & 0 & 0 & 54.8 & 62.5 \\
\hline 10 & 78.0 & 81.5 & 50.7 & 34.4 \\
\hline 11 & $\mathrm{nt}$ & 60.2 & 5.5 & 53.1 \\
\hline
\end{tabular}

${ }^{\mathrm{a}} \mathrm{nt}=$ not tested.

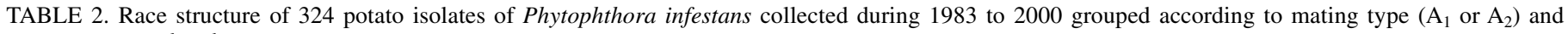
response to metalaxyl

\begin{tabular}{|c|c|c|c|c|c|c|c|c|c|}
\hline \multirow[b]{2}{*}{ Period } & \multicolumn{2}{|c|}{ Virulence phenotype } & \multicolumn{6}{|c|}{ Number of isolates ${ }^{a}$} & \multirow[b]{2}{*}{ Total } \\
\hline & Number & Structures & $\mathrm{A}_{1} \mathrm{~S}$ & $\mathrm{~A}_{1} \mathrm{I}$ & $\mathrm{A}_{1} \mathrm{R}$ & $\mathrm{A}_{2} \mathrm{~S}$ & $\mathrm{~A}_{2} \mathrm{I}$ & $\mathrm{A}_{2} \mathrm{R}$ & \\
\hline \multirow[t]{4}{*}{ 1983-91 } & 3 & 1 & $\ldots$ & $\ldots$ & $\ldots$ & 1 & $\ldots$ & $\ldots$ & 1 \\
\hline & 4 & 1 & $\ldots$ & $\ldots$ & $\ldots$ & 1 & $\ldots$ & $\ldots$ & 2 \\
\hline & 5 & 2 & $\ldots$ & $\ldots$ & $\ldots$ & 10 & $\ldots$ & 9 & 19 \\
\hline & 6 & 1 & $\ldots$ & $\ldots$ & $\ldots$ & 26 & $\ldots$ & 32 & 58 \\
\hline Total & & 5 & $\ldots$ & $\ldots$ & $\ldots$ & 38 & $\ldots$ & 42 & 80 \\
\hline \multirow[t]{6}{*}{ 1993-98 } & 4 & 1 & 1 & $\ldots$ & 1 & 1 & $\ldots$ & $\ldots$ & 3 \\
\hline & 5 & 3 & 10 & $\ldots$ & 1 & 2 & $\ldots$ & $\ldots$ & 13 \\
\hline & 6 & 6 & 12 & 2 & 3 & 2 & $\ldots$ & 1 & 20 \\
\hline & 7 & 6 & 62 & 24 & 27 & 1 & $\ldots$ & 1 & 115 \\
\hline & 8 & 2 & 11 & 2 & 7 & $\ldots$ & $\ldots$ & 1 & 21 \\
\hline & 9 & 1 & 1 & $\ldots$ & $\ldots$ & $\ldots$ & $\ldots$ & $\ldots$ & 1 \\
\hline Total & & 19 & 97 & 28 & 39 & 6 & $\ldots$ & 3 & 173 \\
\hline \multirow[t]{7}{*}{ 1999-2000 } & 3 & 1 & 1 & $\ldots$ & $\ldots$ & $\ldots$ & $\ldots$ & $\ldots$ & 1 \\
\hline & 4 & 4 & 3 & $\ldots$ & 2 & $\ldots$ & $\ldots$ & 1 & 6 \\
\hline & 5 & 10 & 7 & 2 & 1 & 1 & $\ldots$ & 3 & 14 \\
\hline & 6 & 8 & 6 & 3 & 3 & 2 & 3 & 2 & 19 \\
\hline & 7 & 10 & 9 & 3 & 4 & $\ldots$ & 1 & $\ldots$ & 17 \\
\hline & 8 & 6 & 6 & $\ldots$ & 1 & 1 & $\ldots$ & $\ldots$ & 8 \\
\hline & 9 & 3 & 3 & 2 & 1 & $\ldots$ & $\ldots$ & $\ldots$ & 6 \\
\hline Total & $\ldots$ & 42 & 35 & 10 & 12 & 4 & 4 & 6 & 71 \\
\hline
\end{tabular}

a $\mathrm{S}=$ sensitive, $\mathrm{I}=$ intermediate, $\mathrm{R}=$ resistant to metalaxyl. 
(Table 5). Virulence analysis of these isolates on potato differentials showed (Table 5) that only six isolates were simple races carrying three to four virulence factors. The number of isolates carrying five, six, seven, eight, and nine virulence factors was nine, four, seven, four, and two, respectively, with a mean of 5.97 virulence factors per isolate. More than half of the isolates were compatible with the R-9 differential potato. Isolates varied greatly in race structure, with 32 of them belonging to 25 different races (Table 5), thus presenting a mean of 1.3 isolates per race. The frequency of appearance of the 11 virulence factors in the tomato population is given in Table 3. Most frequent were factors 1, 3, 4, 7 , and 9 and least frequent were 2, 5, 6, and 8. Relative to the 1999 to 2000 potato population, a lower frequency of factor 8 and a higher frequency of factor 11 was observed (Table 3 ) in the tomato population. Of the 25 tomato races, 11 also were common to the potato population, whereas 14 races were specific to tomato.

Compatibility of potato and tomato isolates with tomato. Three tomato differential cultivars, ZH, New-Yorker, and Piera-

TABLE 4. RG-57 DNA fingerprints and allozymes loci of 23 Phytophthora infestans isolates collected from potato crops during 1984 to 1999

\begin{tabular}{|c|c|c|c|c|c|c|c|c|c|c|c|c|c|c|c|}
\hline \multirow[b]{2}{*}{ Year } & \multirow[b]{2}{*}{ Isolate } & \multirow[b]{2}{*}{ Type $^{\text {b }}$} & \multirow[b]{2}{*}{$n$} & \multicolumn{8}{|c|}{ DNA bands ${ }^{\mathrm{a}}$} & \multirow[b]{2}{*}{ Octal } & \multirow[b]{2}{*}{ Pep } & \multirow[b]{2}{*}{ Gpi } & \multirow[b]{2}{*}{ Lineage } \\
\hline & & & & 123 & 456 & 789 & 101112 & 131415 & 161718 & 192021 & 22232425 & & & & \\
\hline $1984-86$ & $\ldots$ & $\mathrm{A}_{2} \mathrm{~S}$ & 4 & 100 & 110 & 000 & 100 & 110 & 100 & 011 & 0011 & $130,131,641^{\mathrm{c}}$ & $100 / 100$ & $100 / 100$ & PO-57 \\
\hline $1984-86$ & $\ldots$ & $\mathrm{A}_{2} \mathrm{R}$ & 5 & 100 & 110 & 000 & 100 & 110 & 100 & 011 & 0011 & $130,131,641$ & $100 / 100$ & $100 / 100$ & PO-57 \\
\hline 1993 & $\mathrm{KS}$ & $\mathrm{A}_{1} \mathrm{~S}$ & 1 & 101 & 011 & 111 & 100 & 110 & 100 & 111 & 1011 & $567,131,751^{\mathrm{d}}$ & $100 / 100$ & $100 / 100$ & RFO-39 \\
\hline 1993 & RE & $\mathrm{A}_{2} \mathrm{R}$ & 1 & 101 & 010 & 000 & 100 & 110 & 100 & 111 & 1011 & $520,131,751$ & $100 / 100$ & $100 / 100$ & Unknown \\
\hline 1994 & $\mathrm{EH}$ & $\mathrm{A}_{2} \mathrm{R}$ & 1 & 100 & 011 & 000 & 000 & 110 & 100 & 000 & 1011 & $160,031,051$ & $100 / 100$ & $100 / 100$ & Unknown \\
\hline 1995 & $\mathrm{EHF}$ & $\mathrm{A}_{2} \mathrm{~S}$ & 1 & 100 & 011 & 000 & 000 & 110 & 100 & 001 & 1011 & $160,031,451$ & $100 / 100$ & $100 / 100$ & Unknown \\
\hline 1997 & GED & $\mathrm{A}_{1} \mathrm{R}$ & 1 & 101 & 011 & 111 & 100 & 110 & 100 & 111 & 1011 & $567,131,751$ & $92 / 100$ & $100 / 100$ & RFO-39 \\
\hline 1998 & BIU & $\mathrm{A}_{1} \mathrm{I}$ & 7 & 101 & 011 & 111 & 100 & 110 & 100 & 111 & 1011 & $567,131,751$ & $92 / 100$ & $100 / 100$ & RFO-39 \\
\hline 1999 & BIU & $\mathrm{A}_{1} \mathrm{I}$ & 1 & 101 & 011 & 111 & 100 & 110 & 100 & 111 & 1011 & $567,131,751$ & $\mathrm{nd}^{\mathrm{e}}$ & nd & RFO-39 \\
\hline 1999 & BIU & $\mathrm{A}_{2} \mathrm{I}$ & 1 & 101 & 011 & 111 & 100 & 110 & 100 & 111 & 1011 & $567,131,751$ & nd & nd & RFO-39 \\
\hline
\end{tabular}

a $0=$ negative (no band); 1 = positive.

b $\mathrm{S}=$ sensitive, $\mathrm{R}=$ resistant, $\mathrm{I}=$ intermediate.

${ }^{c}$ Determined by Goodwin to belong to PO-57 found in Poland and Russia $(8,13)$.

d Determined by D. Shaw to belong to RFO-39, common in the United Kingdom and Western Europe (D. Shaw, personal communication).

e nd $=$ not determined.

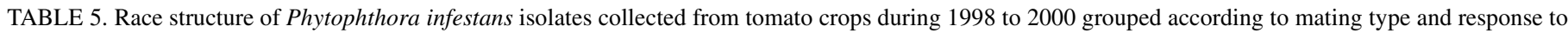
metalaxyl ${ }^{\mathrm{a}}$

\begin{tabular}{|c|c|c|c|c|c|c|c|c|}
\hline \multirow[b]{2}{*}{ Phenotype no., structure ${ }^{b}$} & \multicolumn{2}{|c|}{1998} & \multicolumn{4}{|c|}{1999} & \multirow{2}{*}{$\frac{2000}{\mathrm{~A}_{1} \mathrm{~S}}$} & \multirow[b]{2}{*}{ Total } \\
\hline & $\mathrm{A}_{1} \mathrm{~S}$ & $\mathrm{~A}_{2} \mathrm{~S}$ & $\mathrm{~A}_{1} \mathrm{~S}$ & $\mathrm{~A}_{1} \mathrm{I}$ & $\mathrm{A}_{1} \mathrm{R}$ & $\mathrm{A}_{2} \mathrm{~S}$ & & \\
\hline $\begin{array}{l}\text { Unknown } \\
3\end{array}$ & $\cdots$ & 2 & 1 & $\cdots$ & $\cdots$ & $\ldots$ & $\cdots$ & 3 \\
\hline $4^{134}$ & $\cdots$ & $\cdots$ & $\cdots$ & $\cdots$ & $\cdots$ & 1 & $\cdots$ & 1 \\
\hline $\begin{array}{llll}1 & 3 & 4 & 7 \\
1 & 3 & 4 & 9 \\
1 & 3 & 4 & 10 \\
1 & 3 & 7 & 9\end{array}$ & $\begin{array}{l}1 \\
1 \\
\cdots \\
\cdots\end{array}$ & $\begin{array}{l}\cdots \\
\cdots \\
\cdots \\
\cdots\end{array}$ & $\begin{array}{l}\cdots \\
\cdots \\
\cdots \\
\cdots\end{array}$ & $\begin{array}{l}\cdots \\
\cdots \\
\cdots \\
\cdots\end{array}$ & $\begin{array}{c}1 \\
\cdots \\
\cdots \\
\cdots\end{array}$ & $\begin{array}{l}\cdots \\
\cdots \\
\cdots \\
\cdots\end{array}$ & $\begin{array}{l}\cdots \\
\cdots \\
1 \\
1\end{array}$ & $\begin{array}{l}2 \\
1 \\
1 \\
1\end{array}$ \\
\hline 5 & & & & & & & & \\
\hline $\begin{array}{lllll}1 & 3 & 4 & 5 & 7 \\
1 & 3 & 4 & 5 & 10 \\
1 & 3 & 4 & 7 & 9 \\
1 & 3 & 4 & 7 & 10 \\
1 & 3 & 4 & 10 & 11 \\
1 & 3 & 5 & 7 & 9\end{array}$ & $\begin{array}{l}\cdots \\
\cdots \\
2 \\
\cdots \\
\cdots \\
\cdots\end{array}$ & $\begin{array}{l}\cdots \\
\cdots \\
\cdots \\
\cdots \\
\cdots \\
\cdots\end{array}$ & $\begin{array}{c}\cdots \\
1 \\
1 \\
1 \\
\ldots \\
\ldots\end{array}$ & $\begin{array}{l}1 \\
\cdots \\
\cdots \\
\cdots \\
\cdots \\
\cdots\end{array}$ & $\begin{array}{l}\cdots \\
\cdots \\
\cdots \\
\cdots \\
\cdots \\
\cdots\end{array}$ & $\begin{array}{l}\cdots \\
\cdots \\
\cdots \\
\cdots \\
1 \\
\cdots\end{array}$ & $\begin{array}{c}\cdots \\
\cdots \\
1 \\
\cdots \\
\cdots \\
1\end{array}$ & $\begin{array}{l}1 \\
1 \\
4 \\
1 \\
1 \\
1\end{array}$ \\
\hline 6 & & & & & & & & \\
\hline $\begin{array}{llll}13479 & 41 \\
1347 & 1011 \\
7 & & & \end{array}$ & $\begin{array}{c}1 \\
\ldots\end{array}$ & $\begin{array}{l}\cdots \\
\cdots\end{array}$ & $\begin{array}{l}\cdots \\
\cdots\end{array}$ & $\begin{array}{l}\cdots \\
\ldots\end{array}$ & $\begin{array}{l}\cdots \\
\cdots\end{array}$ & $\begin{array}{l}\ldots \\
1\end{array}$ & $\begin{array}{c}2 \\
\cdots\end{array}$ & $\begin{array}{l}3 \\
1\end{array}$ \\
\hline $\begin{array}{lllllll}1 & 2 & 3 & 6 & 7 & 9 & 11 \\
1 & 2 & 4 & 6 & 7 & 9 & 11 \\
1 & 3 & 4 & 5 & 7 & 10 & 11 \\
1 & 3 & 4 & 6 & 7 & 8 & 9 \\
1 & 3 & 4 & 6 & 7 & 9 & 11 \\
1 & 3 & 4 & 7 & 8 & 9 & 11\end{array}$ & \begin{tabular}{l|}
$\cdots$ \\
$\cdots$ \\
$\cdots$ \\
$\cdots$ \\
$\cdots$ \\
$\cdots$
\end{tabular} & $\begin{array}{l}\cdots \\
\ldots \\
\cdots \\
\cdots \\
\cdots \\
\ldots\end{array}$ & $\begin{array}{c}\ldots \\
1 \\
\ldots \\
1 \\
2 \\
\ldots\end{array}$ & $\begin{array}{l}\cdots \\
\cdots \\
\cdots \\
\cdots \\
\cdots \\
\cdots\end{array}$ & $\begin{array}{l}\cdots \\
\cdots \\
\cdots \\
\cdots \\
\cdots \\
\cdots\end{array}$ & $\begin{array}{l}\cdots \\
\ldots \\
1 \\
\cdots \\
\cdots \\
\ldots\end{array}$ & $\begin{array}{c}1 \\
\cdots \\
\cdots \\
\cdots \\
\cdots \\
1\end{array}$ & $\begin{array}{l}1 \\
1 \\
1 \\
1 \\
2 \\
1\end{array}$ \\
\hline 8 & & & & & & & & \\
\hline $\begin{array}{llllll}1 & 2 & 3 & 457 & 10 & 11 \\
1 & 2 & 4 & 4 & 679 & 11 \\
1 & 4 & 5 & 67 & 10 & 11 \\
1 & 4 & 789 & 10 & 11\end{array}$ & $\begin{array}{l}\cdots \\
\cdots \\
\cdots \\
\cdots\end{array}$ & $\begin{array}{l}\cdots \\
\cdots \\
\cdots \\
\cdots\end{array}$ & $\begin{array}{c}1 \\
1 \\
\cdots \\
\cdots\end{array}$ & $\begin{array}{l}\cdots \\
\cdots \\
\cdots \\
\cdots\end{array}$ & $\begin{array}{l}\cdots \\
\cdots \\
\cdots \\
\cdots\end{array}$ & $\begin{array}{l}\cdots \\
1 \\
1 \\
\cdots\end{array}$ & $\begin{array}{c}\cdots \\
\cdots \\
1\end{array}$ & $\begin{array}{l}1 \\
1 \\
1 \\
1\end{array}$ \\
\hline 9 & & & & & & & & \\
\hline $\begin{array}{l}13457891011 \\
13467891011\end{array}$ & $\begin{array}{l}\cdots \\
\cdots\end{array}$ & $\begin{array}{l}\ldots \\
\ldots\end{array}$ & $\begin{array}{c}\ldots \\
1\end{array}$ & $\begin{array}{l}\ldots \\
\ldots\end{array}$ & $\begin{array}{l}\ldots \\
\ldots\end{array}$ & $\begin{array}{l}\cdots \\
\cdots\end{array}$ & $\begin{array}{c}1 \\
\ldots\end{array}$ & $\begin{array}{l}1 \\
1\end{array}$ \\
\hline Total isolates & 5 & $(2)$ & $10(+1)$ & 1 & 1 & 5 & 10 & 35 \\
\hline Virulence phenotypes & 4 & $\ldots$ & 10 & $\ldots$ & $\ldots$ & 5 & 9 & $\ldots$ \\
\hline Isolates/phenotype & 1.2 & $\ldots$ & 1.0 & $\ldots$ & $\ldots$ & 1.0 & 1.1 & $\ldots$ \\
\hline
\end{tabular}

a $\mathrm{S}=$ sensitive, $\mathrm{I}=$ intermediate, $\mathrm{R}=$ resistant.

b Virulence phenotype number and structure. 
line, carrying genes $P h-O, P h-1$, and $P h-2$, respectively, for resistance against tomato late blight, were inoculated with each of 25 potato or 22 tomato isolates. Results indicate a large difference between the two populations (Table 6). Whereas one, two, and six potato isolates were incompatible with $P h-0, P h-1$, and $P h-2$, respectively (produced hypersensitive necrotic lesions with no sporulation), none of the tomato isolates was incompatible with any of the tomato lines. All tomato isolates were highly compatible with $P h-O$ and $P h-1$, whereas potato isolates were moderately compatible with the two lines carrying these genes. All isolates had a reduced compatibility with $P h-2$, much more so for the potato than for the tomato isolates (Table 6).

A summary of the major traits of $P$. infestans in Israel during 1983 to 2000 is given in Table 7.

\section{DISCUSSION}

We show here that three major waves of genetic changes have taken place during the past 18 years in the population of $P$. infestans in Israel.

The first major change occurred in 1983 , when the $\mathrm{A}_{2}$ mating type was first detected (17) in the country. The $A_{2}$ mating type dominated the pathogen population for the next 9 years. In 1982, the first metalaxyl-resistant (R) isolates were detected (4). The $\mathrm{A}_{2}$ mating type, naturally occurring in Mexico (29), was first reported in the western Hemisphere in 1980 (18). The appearance of $A_{2}$ in Israel has probably resulted from the panglobal distribution of the $A_{2}$ mating type after it was released in $1976(9,13)$ from Mexico to Europe. Israel has no potato or tomato trade with its neighboring countries, Egypt, Jordan, or Syria, indicating that migration of $P$. infestans from these countries is unlikely. The intensive annual potato seed tuber import to Israel from Western Europe may explain the "landing" of $\mathrm{A}_{2}$ in Israel. However, whereas the mean frequency of $\mathrm{A}_{2}$ in European countries ranged between $\approx 20$ and $30 \%$ (11), it was constantly stable at a frequency of $100 \%$ in Israel (except 98\% in 1986) until 1991, indicating that either only the $A_{2}$ genotype was transported from Europe or, if both $A_{1}$ and $A_{2}$ were transported, the $A_{1}$ was much inferior to $A_{2}$ under the Israeli conditions and did not survive. It seems imperative also to assume that the introduced $\mathrm{A}_{2}$ genotype had a much higher fitness than the population (probably $A_{1}$ ) occurring in Israel prior to 1983 , so as to strongly suppress it.
Before the 1980s, worldwide populations of P. infestans were dominated by a single clonal lineage, the US-1 genotype or Ib mitochondrial DNA (mtDNA) haplotype (30). This lineage has since been displaced by the other known modern haplotypes Ia, IIa, and IIb (30). A subset of 27 Israeli isolates collected during 1984 to 2000 were found to belong to Ia (41\%), IIa (56\%), and IIb (3\%) (data not shown), thus reaffirming the possible displacement of the Israeli population during the late 1980s.

In spite of its high fitness, the frequency of isolates belonging to the $\mathrm{A}_{2}$ mating type sharply declined in 1992 to 1993 , until it was totally undetectable by 1996 . A partial reemergence of $\mathrm{A}_{2}$ isolates occurred in 1997 to 2000. Isolates collected during January to April 2001 were all $\mathrm{A}_{1}$ (data not shown). A similar shift from $\mathrm{A}_{2}$ to $\mathrm{A}_{1}$ was reported in Sinaloa, Mexico (19).

The reemergence of $A_{1}$ in 1992 to 1993 may have been another new introduction from Europe, unless we assume that the local $\mathrm{A}_{2}$ changed by selfing to $A_{1}(26)$. The new $A_{1}$ must have had a higher fitness than the predominating $A_{2}$ it had replaced. Indeed, as mentioned above, although $\mathrm{A}_{1}$ and $\mathrm{A}_{2}$ cosurvived in Europe since the $1980 \mathrm{~s}$, the $\mathrm{A}_{2}$ frequency ranged between 20 and $30 \%$ and never exceeded 44\% (in Poland in 1989) $(11,34,37)$ and declined toward 1995 in most European countries (11).

Goodwin et al. found that nine $\mathrm{A}_{2}$ potato isolates he obtained from our 1984 to 1986 potato collection (12) belonged to the PO57 lineage prevailing in Poland and Russia (13). PO-57 is $\mathrm{A}_{2}$, has DNA fingerprinting (octal 130, 131, 641) and allozymes identical to our isolates, but was first observed in Poland much later (in 1990) than in Israel (34). Interestingly, isolates collected in Estonia (34) in 1983 (lineage PO-4) show DNA fingerprinting similar to our 1984 to 1986 isolates (except one band difference) but are $\mathrm{A}_{1}$.

Parallel changes were observed in the response of $P$. infestans to metalaxyl. Resistance was first observed in 1982 (4). During 1983 to 1991 , when all isolates were $A_{2}$, the mean frequency of metalaxyl-resistant versus metalaxyl-sensitive isolates was 67 and $33 \%$, respectively, with resistant isolates being much fitter to potato than sensitive ones $(22,24)$. The first appearance of metalaxylintermediate isolates occurred in 1993 (no data available for 1992), concurrent with the reemergence of $A_{1}$ isolates.

Although oospores may develop in potato crops in Israel (2), it is not clear if this appearance of I isolates was a consequence of local mating ( $\mathrm{I}$ isolates are often offspring of sexual mating

TABLE 6. Compatibility of potato and tomato isolates of Phytophthora infestans with tomato differential cultivars carrying genes for resistance against late blight $^{\mathrm{a}}$

\begin{tabular}{|c|c|c|c|c|c|c|c|c|c|c|}
\hline \multirow[b]{3}{*}{ Tomato differential } & \multicolumn{10}{|c|}{ Proportion of isolates showing compatibility index ${ }^{b}$} \\
\hline & \multicolumn{5}{|c|}{ Potato isolates $(n=25)^{\mathrm{c}}$} & \multicolumn{5}{|c|}{ Tomato isolates $(n=22)^{\mathrm{d}}$} \\
\hline & HR & 1 & 2 & 3 & 4 & HR & 1 & 2 & 3 & 4 \\
\hline Ph-1 (NewYorker) & 8 & 8 & 16 & 16 & 52 & 0 & 0 & 0 & 0 & 100 \\
\hline Ph-2 (Pieraline) & 24 & 16 & 40 & 16 & 0 & 0 & 18 & 68 & 14 & 0 \\
\hline
\end{tabular}

${ }^{\mathrm{a}}$ Tests were conducted with detached leaflets floating on water at $17^{\circ} \mathrm{C}$.

b The 1-to-4 scale represents the lesion size and abundance of sporangial production at 7 days postinoculation, where HR = hypersensitive response, dark brown lesions with no sporulation; 1 = lesions, 5 to $10 \mathrm{~mm}$ in diameter, with scarce sporulation; $2=$ lesions, 10 to 15 mm in diameter, with moderate sporulation; 3 = lesions, 15 to $20 \mathrm{~mm}$ in diameter, with abundant sporulation; and 4 = lesions, 20 to $25 \mathrm{~mm}$ in diameter, with abundant sporulation.

c Isolates collected during 1993 to 2000.

d Isolates collected during 1997 to 2000

TABLE 7. Summary of the characteristic of Phytophthora infestans in Israel during 1983 to $2000^{\mathrm{a}}$

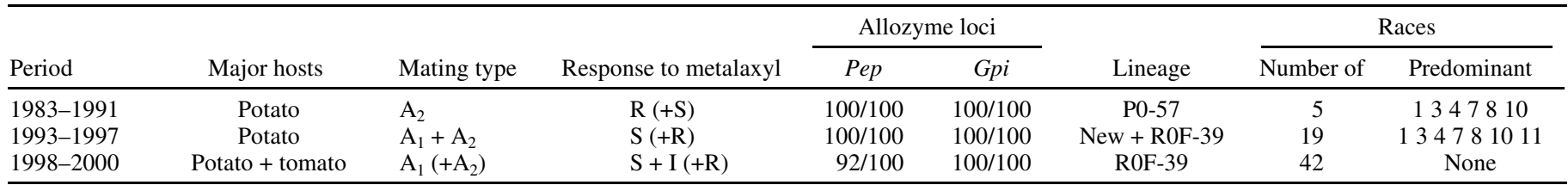

${ }^{a}$ Figures in parenthesis indicate a low proportion in the population. S, I, and R = sensitive, intermediately resistant, and resistant to metalaxyl, respectively. 
between $S$ and $R$ isolates) $(11,20,32)$ because little $A_{1}$ occurred in the country to enable such mating (unless we assume that $A_{1}$ had already reemerged in 1992). Intermediate isolates which were rare in Switzerland, for example, in 1988 to 1990 were doubled in 1992 to 1993 and further increased to $30 \%$ in 1994 to 1995 (11). It seems, therefore, that the $\mathrm{A}_{1}$, which was reintroduced to Israel in 1992 to 1993, was either metalaxyl-sensitive, -intermediate, or both. S, I, and R subpopulations probably derived from mating between $A_{1}$ and $A_{2}$ isolates whose proportions fluctuated during 1993 to 2000 (Fig. 1B).

Much effort was devoted in this study to determine the virulence phenotypes (race structure) present in the local population of $P$. infestans. In the $1950 \mathrm{~s}$ and $1960 \mathrm{~s}$, race 4 was frequent and, to a lesser extent, races $0,1,3,1.4$, and $1.3 .4(25,36)$. In the $1970 \mathrm{~s}$, race 0 was still present (31).

Isolates we collected from potato during 1983 to 1986 (24) were 1.3.4.7.8 or 1.3.4.7.8.10. During 1987 to 1991 , only five races were found (on nine potato differentials) among 74 isolates collected from potato. Of these, $78 \%$ were $1,3,4,7,8,10$ and the rest carried three to five virulence factors. Kadish (20) made parallel tests (1987 to 1991) with isolates he collected from tubers imported to Israel from western Europe. Amongst 29 isolates, 18 carried two virulence factors and only one isolate from Europe was $1,3,4,7,8,10$. The predominance of this race in Israel during 1987 to 1998 may be attributed to a local evolvement, import, or both. Although potato $R_{11}$ was not included in 1983 to 1986 or in 1987 to 1991 (24), it may be possible that virulence factor 11 was also present in these populations.

Race structure of the 1993 to 1998 populations from potato was mostly $(63 \%)$ composed of virulence factors $1,3,4,7,8,10$, and 11 , with the rest $(37 \%)$ of the population exhibiting 18 other virulence combinations. The predominant race was distributed at a ratio of $2: 1: 1$ among $S / I / R$ isolates of the prevailing $A_{1}$ mating type. The population collected during 1999 to 2000 presented 42 race structures among 71 isolates from potato, with almost every second isolate belonged to another race. Similar variability in genetic structure was observed by U. Gisi (personal communication) for isolates collected in 1996 to 1997 in France and Switzerland. Our races were highly complex, having up to nine virulence factors. The reasons for this complexity are not clear. The fact that $\approx 30$ different cultivars of potato are annually imported from Europe and commercially grown in the country may have contributed to it. The major changes occurred in factors 2, 5, and 9, whose frequencies were increased, as against factors 7, 8, and 10, which have declined. A different situation occurred in Poland, where factor 9 was not detected among 1,177 isolates collected during 1987 to 1998 and factor 5 was present at a frequency of only 5\% (35), or in North America (16), where the older clonal lineages, US-1 and US-6, had much more pathogenic diversity than the more recently immigrated genotypes US-7 and US-8.

A recent study conducted in 1998 to 1999 by Derie and Inglis (5) in western Washington revealed highly complex races in isolates of US-7, US-8, and US-14 genotypes (8 to 9.3 virulence factors). Factor 9 was the rarest (4 of 109 isolates, 3.7\%). These authors indicated that unnecessary virulences have been maintained in the population since 1990. Whether a similar persistence of virulences will hold in Israel remains to be studied in the future.

Of special interest are the characteristics of the tomato isolates. Heavy epidemics of late blight occurred in tomato during 1999 to 2000. Heavy epidemics were recently reported in California (10) and Europe (27). Almost all our isolates $(80 \%)$ were $A_{1}$, thus performing a different situation compared with Europe (27) or the United States (28), where $\mathrm{A}_{2}$ was equally or preferentially isolated from tomatoes. During that period, a similar ratio of $87: 13$ between $A_{1}$ and $A_{2}$ also was recorded in our potato isolates.

The 32 tomato isolates examined were composed of 25 races (on potato differentials), of which 14 were specific to tomato. More than half were complex races containing seven to nine viru- lence factors, unlike tomato isolates in France and Switzerland, which were found to be simple races (U. Gisi, personal communication). The tomato and potato populations collected during 1999 to 2000 showed similar frequencies of virulence factors except factor 8 (which was lower in tomato) and factor 11 (which was higher in tomato). Apparently, factor 11 in tomato showed a frequency $(\approx 60 \%)$ similar to that of the potato population collected earlier (1993 to 1998) (Table 3). Moreover, all our tomato isolates, but not potato isolates, profusely sporulated on leaves of the tomato differentials carrying the genes $P h-O$ or $P h-1$ for resistance against tomato late blight, with none of them showing HR with $P h-2$. Such race complexity and aggressiveness may partially explain the severe epidemics caused by these isolates in tomato crops in Israel. Goodwin et al. (16) showed that most US-1 isolates did not infect tomato. With the widespread distribution of the immigrated US-6 and US-7 genotypes, however, late blight epidemics on potato could serve as a source of inoculum for tomato fields (16). Legard et al. (28) suggested that aggressiveness of $P$. infestans to tomato may be a recently acquired trait. Tomatoaggressive isolates caused severe disease on both potato and tomato, but significantly more disease on tomato than on potato (28). We assume that tomato isolates in our country were locally evolved from the potato population and acquired specialization to tomato. Whether this specialization took place via sexual recombinations is not known.

Taken together, the Israeli population of $P$. infestans varied greatly during the 18 -year period of this study. Major changes have occurred in 1982 to 1983 , when resistance to metalaxyl and $\mathrm{A}_{2}$ were first introduced; in 1992 to 1993 , when the $\mathrm{A}_{2}$ population was replaced by $A_{1}$ while isolates with intermediate sensitivity to metalaxyl first occurred; and in 1999 to 2000, when an $\mathrm{A}_{1}$-sensitive population with extreme virulence variation and high aggressiveness to tomato dominated. These shifts may partially reflect the changes that occurred in Europe, from which potato tubers are annually imported, but also implicate a rapid local evolution of new genotypes.

\section{ACKNOWLEDGMENTS}

I thank the following people for their assistance: O. Pisanti, Y. Samoucha, D. Kadish, S. Farkash, S. Klopman, Y. Kinar, E. Rubin, M. Galperin, A. Baider, D. Shaw, and U. Gisi.

\section{LITERATURE CITED}

1. Andrivon, D. 1995. Biology, ecology and epidemiology of the potato late blight pathogen Phytophthora infestans in soil. Phytopathology 85:1053-1056.

2. Cohen, Y., Farkash, S., Baider, A., and Shaw, D. S. 2000. Sprinkling irrigation enhances production of oospores of Phytophthora infestans in field-grown crops of potato. Phytopathology 90:1105-1111.

3. Cohen, Y., Farkash, S., Reshit, Z., and Baider, A. 1997. Oospore production of Phytophthora infestans in potato and tomato leaves. Phytopathology 87:191-196.

4. Cohen, Y., and Reuveni, M. 1983. Occurrence of metalaxyl-resistant isolates of Phytophthora infestans in potato fields in Israel. Phytopathology 73:925-927.

5. Derie, M. L., and Inglis, D. A. 2001. Persistence of complex virulences in populations of Phytophthora infestans in western Washington. Phytopathology 91:606-612.

6. Drenth, A., Janssen, E. M., and Govers, F. 1995. Formation and survival of oospores of Phytophthora infestans under natural conditions. Plant Pathol. 44:86-94.

7. Drenth, A., Tas, I. C. Q., and Govers, F. 1994. DNA fingerprinting uncovers a new sexually reproducing population of Phytophthora infestans in the Netherlands. Eur. J. Plant Pathol. 100:97-107.

8. Forbes, G. A., Goodwin, S. B., Drenth, A., Oyarzun, P., Ordonez, M. E., and Fry, W. E. 1998. A global marker database for Phytophthora infestans. Plant Dis. 82:811-818.

9. Fry, W. E., Goodwin, S. B., Dyer, A. T., Matuszak, J. M., Drenth, A., Tooley, P. W., Sujkowski, L. S., Koh, Y. J., Cohen, B. A., Spielman, L. J., Deahl, K. L., Inglis, D. A., and Sandlan, K. P. 1993. Historical and recent 
migrations of Phytophthora infestans: Chronology, pathways and implications. Plant Dis. 77:653-661.

10. Gavino, P. D., Smart, C. D., Sandrock, R. W., Miller, J. S., Hamm, P. B., Lee, T. Y., Davis, R. M., and Fry, W. E. 2000. Implications of sexual reproduction for Phytophthora infestans in the United States: Generation of an aggressive lineage. Plant Dis. 84:731-735.

11. Gisi, U., and Cohen, Y. 1996. Resistance to phenylamide fungicides: A case study with Phytophthora infestans involving mating type and race structure. Annu. Rev. Phytopathol. 34:549-572.

12. Goodwin, S. B. 1991. DNA polymorphism in Phytophthora infestans: The Cornell experience. Pages 256-271 in: Phytophthora. J. A. Lucas, R. C. Shattock, D. S. Shaw, and L. R. Cooke, eds. Cambridge University Press, Cambridge.

13. Goodwin, S. B., Cohen, B. A., and Fry, W. E. 1994. Panglobal distribution of a single clonal lineage of the Irish potato famine fungus. Proc. Nat. Acad. Sci. USA 91:11591-11595.

14. Goodwin, S. B., Drenth, A., and Fry, W. E. 1992. Cloning and genetic analyses of two highly polymorphic, moderately repetitive nuclear DNAs from Phytophthora infestans. Curr. Genet. 22:107-115.

15. Goodwin, S. B., Schneider, R. E., and Fry, W. E. 1995. Use of celluloseacetate electrophoresis for rapid identification of allozyme genotypes of Phytophthora infestans. Plant Dis. 79:1181-1185.

16. Goodwin, S. B., Sujkowski, L. S., and Fry, W. E. 1995. Rapid evolution of pathogenicity within clonal lineages of the potato late blight disease fungus. Phytopathology 85:669-676.

17. Grinberger, M., Kadish, D., and Cohen, Y. 1989. Occurrence of the $A_{2}$ mating type and oospores of Phytophthora infestans in potato crops in Israel. Phytoparasitica 17:197-204.

18. Hohl, H. R., and Iselin, K. 1984. Strains of Phytophthora infestans with A2 mating type behaviour. Trans. Br. Mycol. Soc. 83:529-530.

19. Jaime-Garcia, R., Trinidad-Correa, R., Felix-Gastelum, R., Orum, T. V., Wasmann, C. C., and Nelson, M. R. 2000. Temporal and spatial patterns of genetic structure of Phytophthora infestans from tomato and potato in the Del Fuerte Valley. Phytopathology 90:1188-1195.

20. Kadish, D. 1994. Fitness of metalaxyl-sensitive and metalaxyl-resistant isolates of Phytophthora infestans on potato cultivars: Epidemiological and genetical aspects. Ph.D. thesis. Bar-Ilan University, Ramat-Gan, Israel.

21. Kadish, D., and Cohen, Y. 1988. Estimation of metalaxyl-resistance in Phytophthora infestans. Phytopathology 78:915-919.

22. Kadish, D., and Cohen, Y. 1989. Population dynamics of metalaxyl-sensitive and metalaxyl-resistant isolates of Phytophthora infestans in untreated crops of potato. Plant Pathol. 38:271-276.

23. Kadish, D., and Cohen, Y. 1992. Overseasoning of metalaxyl-sensitive and metalaxyl-resistant isolates of Phytophthora infestans in potato tubers. Phytopathology 82:887-889.
24. Kadish, D., Grinberger, M., and Cohen, Y. 1990. Fitness of metalaxylsensitive and metalaxyl-resistant isolates of Phytophthora infestans on susceptible and resistant potato cultivars. Phytopathology 80:200-205.

25. Kedar (Kammermann), N., Rotem, J., and Wahl, I. 1959. Physiologic specialization of Phytophthora infestans in Israel. Phytopathology 49:675-679.

26. Ko, W. H. 1994. An alternative possible origin of the A2 mating type of Phytophthora infestans outside Mexico. Phytopathology 84:1224-1227.

27. Lebreton, L., and Andrivon, D. 1998. French isolates of Phytophthora infestans from potato and tomato differ in phenotype and genotype. Eur. J. Plant Pathol. 104:583-594.

28. Legard, D. E., Lee, T. Y., and Fry, W. E. 1995. Pathogenic specialization in Phytophthora infestans: Aggressiveness on tomato. Phytopathology 85:1356-1361.

29. Niederhauser, J. S. 1991. Phytophthora infestans: The Mexican connection. Pages 25-45 in: Phytophthora. J. A. Lucas, R. C. Shattock, D. S. Shaw, and L. R. Cooke, eds. Br. Mycol. Soc. Cambridge University Press, Cambridge.

30. Ristaino, J. B., Groves, C. T., and Parra, G. R. 2001. PCR amplification of the Irish potato famine pathogen from historic specimens. Nature 411:695-697.

31. Rotem, J., and Cohen, Y. 1974. Epidemiological patterns of Phytophthora infestans under semi-arid conditions. Phytopathology 64:711-714.

32. Shattock, R. C. 1986. Inheritance of metalaxyl resistance in the potato late-blight fungus. Br. Crop Prot. Counc. Brighton Metropole, Brighton, England 2:555-560.

33. Stromberg, A., Peterson, L., and Wikstrom, M. 1999. Infection of potato by oospores of Phytophthora infestans in soil. Plant Dis. 83:876.

34. Sujkowski, L. S., Goodwin, S. B., Dyer, A. T., and Fry, W. E. 1994. Increased genotypic diversity via migration and possible occurrence of sexual reproduction of Phytophthora infestans in Poland. Phytopathology 84:201-207.

35. Swiezynski, K. M., Domanski, L., Zarzycka, H., and ZimnochGuzowska, E. 2000. The reaction of potato differentials to Phytophthora infestans isolates collected in nature. Plant Breed. 119:119-126.

36. Sztenjnberg, A., and Wahl, I. 1966. Studies on the host range of the potato late blight fungus and its oversummering on wild and ornamental Solanaceae plants. Pages 452-460 in: Proc. 1st Congr. Mediterr. Phytopathol. Union. Bari-Naples, Italy.

37. Therrien, C. D., Ritch, D. L., Sujkowski, L. S., Spielman, L. J., Fry, W. E., Daggett, S. S., Sim, J. H., and Tooley, P. W. 1993. Phytophthora infestans in Poland from 1987-1989; nuclear DNA content, mating type distribution and response to metalaxyl. J. Phytopathol. 139:68-80.

38. Zwankhuizen, M. J., Govers, F., and Zadoks, J. C. 2000. Inoculum sources and genotypic diversity of Phytophthora infestans in Southern Flevoland, the Netherlands. Eur. J. Plant Pathol. 106:667-680. 